\title{
Structural Vibration Sensing to Evaluate Animal Activity on a Pig Farm*
}

\author{
Amelie Bonde \\ Carnegie Mellon University \\ Moffett Field, California \\ amelie@cmu.edu
}

Akkarit Sangpetch

King Monkut's Institute of

Technology Ladkrabang

Ladkrabang, Bangkok, Thailand

akkarit@cmkl.ac.th
Extended Abstract

\author{
Shijia Pan \\ Carnegie Mellon University \\ Pittsburgh, Pennsylvania \\ shijiapan@cmu.edu
}
Woranun Woramontri
Betagro Group
Thailand
woranunw@betagro.com

\author{
Pei Zhang \\ Carnegie Mellon University \\ Moffett Field, California \\ peizhang@cmu.edu
}

\author{
Orathai Sangpetch \\ King Monkut's Institute of \\ Technology Ladkrabang \\ Ladkrabang, Bangkok, Thailand \\ orathai@cmkl.ac.th \\ Hae Young Noh \\ Carnegie Mellon University \\ Pittsburgh, Pennsylvania \\ noh@cmu.edu
}

\section{CCS CONCEPTS}

-Human-centered computing $\rightarrow$ Ubiquitous and mobile computing systems and tools; • Computer systems organization $\rightarrow$ Embedded and cyber-physical systems;

ACM Reference Format:

Amelie Bonde, Shijia Pan, Orathai Sangpetch, Akkarit Sangpetch, Woranun Woramontri, Hae Young Noh, and Pei Zhang. 2018. Structural Vibration Sensing to Evaluate Animal Activity on a Pig Farm: Extended Abstract. In The 1st Workshop on Data Acquisition To Analysis (DATA'18), November 4, 2018, Shenzhen, China. ACM, New York, NY, USA, 2 pages. https://doi.org/ $10.1145 / 3277868.3277881$

\section{INTRODUCTION}

Automated monitoring of animal behavior can detect changes in animal welfare and health problems. Different animal behaviors can point to disease, unrest or inadequate management, and detecting such behaviors in real time allows automated monitoring to be a valuable tool in livestock production [2]. When monitoring pigs, important sow events such as oestrus, pregnancy or parturition may also be detected through continuous animal monitoring [1].

Prior work takes a number of approaches, using video or image analysis, infrared detectors, and accelerometers worn by the

\footnotetext{
"Produces the permission block, and copyright information

Permission to make digital or hard copies of all or part of this work for personal or classroom use is granted without fee provided that copies are not made or distributed for profit or commercial advantage and that copies bear this notice and the full citation on the first page. Copyrights for components of this work owned by others than ACM must be honored. Abstracting with credit is permitted. To copy otherwise, or republish, to post on servers or to redistribute to lists, requires prior specific permission and/or a fee. Request permissions from permissions@acm.org.

DATA'18, November 4, 2018, Shenzhen, China

(c) 2018 Association for Computing Machinery.

ACM ISBN 978-1-4503-6049-4/18/11.

https://doi.org/10.1145/3277868.3277881
}

pigs $[1,4,6]$. The studies using video, image processing, and infrared measure activity level or activity index, while Escalante et. al. do activity classification on several types of pig activity using pig-mounted accelerometers.

We propose using geophones attached to the underside of the floor of a pig pen to sense the vibrations generated by the pigs. We hope that our vibration-based method will allow for both measuring how much activity is happening and what types of activities those might be. Vibration sensors mounted in the floor are less intrusive than wearable sensors, and unlike most video cameras, they don't require light to operate. Vibration sensing from geophones has been successful in many human monitoring scenarios such as identification [7], localization [5], and health tracking [3].

\section{HARDWARE SETUP}

Our hardware setup uses geophone sensors. Geophones are widely used for seismic sensing and structural health monitoring because of their sensitivity to ground movement. A geophone consists of a spring-mounted magnet that moves within a wire coil to generate a voltage, which can thus measure movement at different frequencies. Its has low noise and high sensitivity at frequencies of $7 \mathrm{~Hz}$ and above [3]. The geophone we use, SM-24 Geophone Elements [8] has a natural frequency of $10 \mathrm{~Hz}$. We install the geophone with an amplifier and a high pass filter.

Because the floors of a pig pen become covered in excrement and are regularly hosed down with water, the sensors need to be protected. We enclose the electronics in waterproof boxes designed to NEMA $4 \mathrm{x}$ specifications, which are suited for this environment. We connect the geophones to the boxes with insulated wire through waterproof connectors. Power is provided through cables attached in the same manner. We superglue the box and geophone to the underside of the floor of the pen, and use insulating foam to further protect them from the elements. 


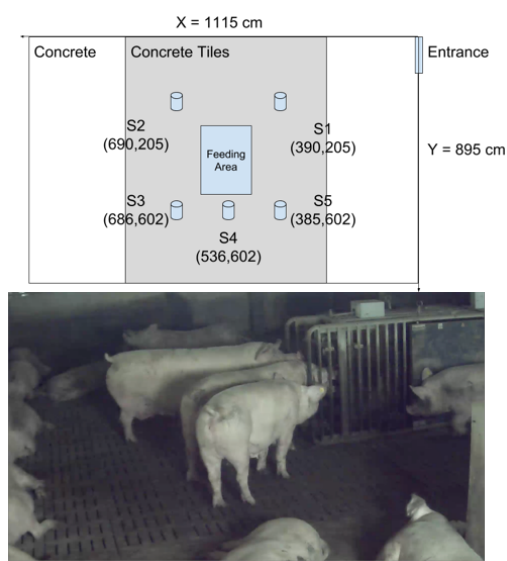

Figure 1: A diagram of sensor placement in the group pen, and view from the ground truth camera. All measurements are in $\mathbf{c m}$.

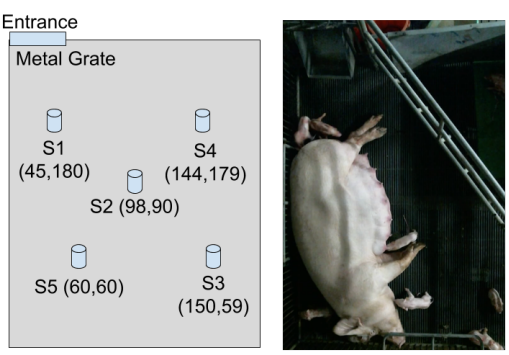

Figure 2: A diagram of sensor placement in the individual pen, and view from the ground truth camera. All measurements are in $\mathrm{cm}$.

\section{EXPERIMENTS}

We deployed our system at Betagro Farm near Lopburi, Thailand. We installed the sensors in two locations: an individual pen designed for a single sow and her piglets, and a group pen designed for 20-30 pigs. Each pen has five sensors installed on the underside of the floor of the pen, as shown in the diagrams of Figure 1 and Figure 2. A video camera aimed at each pen collects ground truth data of the pigs' activities. The data is time stamped when it arrives at the server.

\subsection{Data File Settings}

The data is organized by location and day. Each location folder contains a folder for each day, and each day folder contains a folder of video files, labeled by time, and a folder of text files containing the geophone data. The geophone data is collected in one file per sensor. Each text file is a list of JSON arrays with all the data packets sent over the course of the day. Each data packet contains metadata and 1000 data samples sampled at $2000 \mathrm{~Hz}$, so a half second of data. Below is an example of one JSON array, with example values used and the data replaced with "..." for brevity:

\{

"_id" : ObjectId("5b575b13084d730006e6660e"),

"_class" : "org.json.simple.JsonObject",

"lng" : 100.777404,

"data" : "[...]",

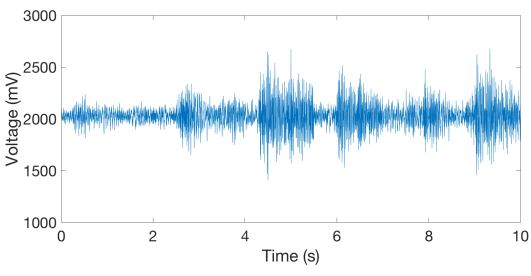

Figure 3: 10 seconds of raw data from sensor 2 in the individual pen.

"uuid" : "Geophone_67F5B9",

"lat" : 13.727277,

"ts" : NumberLong("1532451600464"),

"timestamp" : NumberLong("1532451600464")

\}

The timestamp is Unix epoch time in milliseconds.

\section{CONCLUSION}

We deployed vibration sensors in two different pens at Betagro pig farm in Lopburi, Thailand. We provide a sample of the data in this continuously running experiment, and will provide more data as the deployment progresses. Our hope is that researchers will use this data set to detect important pig events as well as changes in animal health and welfare.

\section{ACKNOWLEDGEMENTS}

This research was supported in part by the National Science Foundation Graduate Research Fellowship Program (under Grant No. DGE 1745016), Carnegie Mellon UniversityâĂŹs Mobility21 National University Transportation Center (grant number 69A3551747111), Intel, Google and AiFi. The views and conclusions contained here are those of the authors and should not be interpreted as necessarily representing the official policies or endorsements, either express or implied, of Carnegie Mellon University, the National Science Foundation, or the U.S. Government or any of its agencies.

\section{REFERENCES}

[1] Hugo Jair Escalante, Sara V Rodriguez, Jorge Cordero, Anders Ringgaard Kristensen, and Cécile Cornou. 2013. Sow-activity classification from acceleration patterns: a machine learning approach. Computers and electronics in agriculture 93 (2013), 17-26.

[2] AR Frost, CP Schofield, SA Beaulah, TT Mottram, JA Lines, and CM Wathes. 1997. A review of livestock monitoring and the need for integrated systems. Computers and electronics in agriculture 17, 2 (1997), 139-159.

[3] Zhenhua Jia, Amelie Bonde, Sugang Li, Chenren Xu, Jingxian Wang, Yanyong Zhang, Richard E Howard, and Pei Zhang. 2017. Monitoring a Person's Heart Rate and Respiratory Rate on a Shared Bed Using Geophones. In Proceedings of the 15th ACM Conference on Embedded Network Sensor Systems. ACM, 6.

[4] Mohammad Amin Kashiha, Claudia Bahr, Sanne Ott, Christel PH Moons, Theo A Niewold, Frank Tuyttens, and Daniel Berckmans. 2014. Automatic monitoring of pig locomotion using image analysis. Livestock Science 159 (2014), 141-148.

[5] M. Mirshekari, S. Pan, J. Fagert, E.M. Schooler, P. Zhang, and H.Y. Noh. 2018. Occupant localization using footstep-induced structural vibration. Mechanical Systems and Signal Processing 112 (2018), 77-97.

[6] Sanne Ott, CPH Moons, Mohammadamin A Kashiha, Claudia Bahr, FAM Tuyttens, Daniel Berckmans, and Theo A Niewold. 2014. Automated video analysis of pig activity at pen level highly correlates to human observations of behavioural activities. Livestock Science 160 (2014), 132-137.

[7] S. Pan, T. Yu, M. Mirshekari, J. Fagert, A. Bonde, O. J. Mengshoel, and P. Zhang. 2017. FootprintID: Indoor Pedestrian Identification through Ambient Structural Vibration Sensing. Proceedings of the ACM on Interactive, Mobile, Wearable and Ubiquitous Technologies 1, 3 (2017), 89.

[8] Sparkfun. 2017. Geophone SM-24. https://www.sparkfun.com/products/11744 